\title{
Cri du chat syndrome: A critical review
}

\author{
Ángela Rodríguez-Caballero ${ }^{1}$, Daniel Torres-Lagares ${ }^{2}$, Antonio Rodríguez-Pérez ${ }^{1}$, María-Ángeles Serrera- \\ Figallo $^{3}$, José-María Hernández-Guisado ${ }^{3}$, Guillermo Machuca-Portillo ${ }^{4}$
}

\footnotetext{
${ }^{1}$ Doctoral Fellow. Master in Integrated Dentistry of Adults and Special Patients. Departament of Dentistry. University of Sevilla

${ }^{2}$ Regular Doctor Proffesor. Oral Surgery. Departament of Dentistry. University of Sevilla

${ }^{3}$ Associated Proffesor. Integrated Dentistry of Adults and Special Patients. Departament of Dentistry. University of Sevilla

${ }^{4}$ Titular Proffesor. Director of Master in Integrated Dentistry of Adults and Special Patients. Departament of Dentistry. University of Sevilla
}

\author{
Correspondence: \\ Facultad de Odontología de Sevilla \\ Hospital Virgen del Rocío \\ C/ Avicena $s / n$ \\ 41009 Sevilla, Spain \\ gmachuca@us.es
}

Received: 22/03/2009

Accepted: 20/09/2009

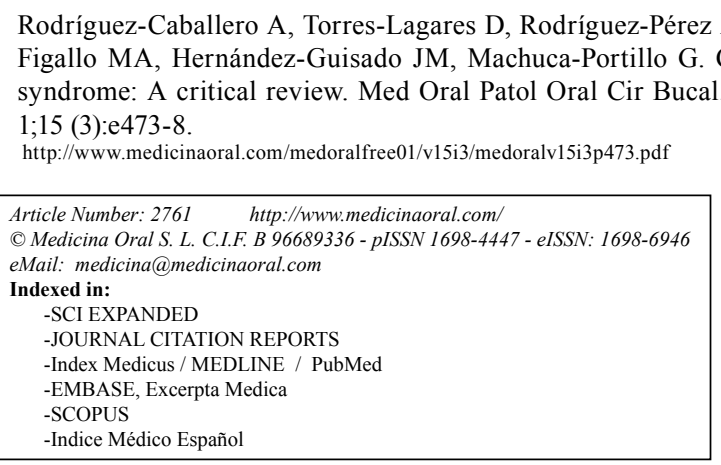

\begin{abstract}
A new syndrome was identified in 1963, when Lejeune et al. reported a genetic disease resulting from a partial or total deletion on the short arm of chromosome 5 (5p-) and named it the cri du chat syndrome (CdCS). This term makes reference to the main clinical feature of the syndrome, a high-pitched monochromatic cat-like crying, that usually disappears in the first years of life.

$\mathrm{CdCS}$ is one of the most common chromosomal deletion syndromes in humans, with an incidence of 1:15.000-1:50.000 live-births.

Our purpose was to review different aspects of this syndrome (concept, epidemiology, aetiology, clinical features, diagnostic methods and prognosis) emphasizing both: the breakthrough in this field introduced by new cytogenetic and molecular techniques, and the orofacial manifestations most frequently reported.

The main orofacial anomalies registered were mandibular microretrognathia, high palate but rarely cleft, variable malocclusion (frequently anterior open-bite), enamel hypoplasia, poor oral hygiene, generalized chronic periodontitis, and retardation of tooth eruption, although there is not enough evidence to support any clear association between these pathologies and the CdCS.
\end{abstract}

Key words: Cri du chat syndrome, chromosome disorders, orofacial manifestation. 


\section{Definition}

The Cry du Chat Syndrome (CdCS) was first described by Lejeune et al. (1) in 1963. The name of the syndrome refers to the most characteristic clinical feature, a highpitched crying similar to the mewing of a cat, which usually disappears in the first years of life.

The syndrome results from a total or partial deletion on the short arm of the chromosome 5 (5p-) (range from $35 \%$ to $55 \%$ ) being associated with a de novo deletion in more than 80 percent of the cases (2).

In recent years, the application of genetic molecular methods introduced advances in the diagnosis and typification of the CdCS. Our purpose is to update the knowledge in this direction, and to review the main clinical features and oral manifestations in this syndrome that requires special odontological management.

\section{Epidemiology}

Despite being considered a rare genetic autosomal disorder, $\mathrm{CdCS}$ is one of the most common chromosomal syndromes in humans (3). The first epidemiological studies estimated the prevalence at 1 in 50.000 live-births, nevertheless subsequent studies suggested a greater prevalence at 1 in 37.000 live-born infants (3). The incidence in females is slightly higher $(1,3)$, however significant differences in the distribution of CdCS among races or geographical areas has not been established.

Among the mentally retarded population CdCS represents less than 1 percent. Concerning this subject of study, Niebuhr (3) reported a proportion of 1:350 between 6.000 individuals with a mental handicap.

Likewise, any outstanding association with prenatal events, age of parents, or location according to order of birth within the family has not been established (3). A survey of 27 mothers made by Cornish y Pigram (1996) showed that $37 \%$ had suffered some kind of anomaly during the pregnancy. The labor had been complicated in $44 \%$ of the cases (4). Occasionally, parental exposition to radiation and maternal disease, including hyperemesis, anorexia, toxemia and vaginal bleeding were reported (3).

\section{Etiopathogenesis}

Like it was described by Lejeune et al. in 1963, the $\mathrm{CdCS}$ is a dysmorphogenic syndrome consisting in a chromosomopathy caused by a partial or total deletion of genetic material on the short arm of the chromosome 5. The size of the deletion could affect from region $5 p$ 15.3 to the complete loss of the short arm.

Most of the cases are due to a de novo deletion (around $80 \%$ ), a little more than $10 \%$ of the cases are originated by a parental translocation, and less than $10 \%$ are associated with cytogenetic rare aberrations (3). By evaluating a sample of 80 patients and 148 parents Mainardi et al. (2) discovered that $77.5 \%$ of the patients showed a terminal deletion; $8.75 \%$ an interstitial deletion; $5 \%$ a de novo translocation; $3.75 \%$ a familiar translocation; $3.75 \%$ a mosaic with two cellular lines and the $1 \%$ remaining showed a pericentric inversion. This deletion often has a paternal origin (80-90\% of the cases) (2).

Niebuhr was the first one to identify the specific chromosomal region involved in CdCS (5p 15.1-5p 15.3) through cytogenetic analysis. Thanks to the breakthrough introduced by cytogenetic molecular analysis based on in situ hybridization (FISH) we have been able to develop more precise studies in which areas within this region related to certain clinical features of the syndrome have been identified (2). The breakpoints have a range from p13 to p15.2 (2), and within this interval we can distinguish two regions; one associated with the typical crying of the CdCS (located in 5p 15.3), and the other associated with dysmorphism, microcephaly and mental handicap (located in 5p 15.2) (2).

\section{Clinical Features}

\subsection{Neonatal period}

During the neonatal period the typical cry is probably the most characteristic and striking clinical feature in newborns and young children. It is a weak, monotonous, high-pitched crying similar to the mewing of a cat (1) that usually disappears within a few months or years of life, however the duration of its existence is controversial.

As an exception, isolated atypical cases have been registered where the crying was not present or instead there was a persistent stridor during the inspiration. Nevertheless, we could not consider it a pathognomonical sign of the syndrome, because it has also been found in certain kinds of disorders or neurologic diseases (3).

The pathogenesis of this crying was attributed to morphologic laryngeal alterations by the first published cases (1): flappy, small and curved epiglottis, hypoplastic, narrow and diamond-shaped or quadrangular larynx, having an abnormal air space in the posterior area during phonation $(5,6)$. Nevertheless, subsequent studies established that these anatomical alterations were not necessarily present in every patient, therefore the existence of some other organic and/or functional factors were not being considered (5). Currently the pathogenesis is associated not only with anatomical alterations in the epiglottis and the larynx but also with structural and/or functional neurological alterations $(5,6)$.

In addition to the crying, the newborn with CdCS presents a low birth weight (below 20th percentile, $2.614 \mathrm{~g}$ average weight), and small size (always below 50th percentile) despite a gestational age close to the norm (5).

During the first two years of life, their growth and development is remarkably slow because of the feeding difficulties (weak and impaired suction, dysphagia, muscle hypotonia, gastroesophageal and nasal reflux) (5). 
Other early and frequent complications are asphyxia episodes, cyanotic crisis and inspiratory stridor (5). Furthermore, recurrent respiratory infections and, less frequently, bronchiectasis associated with pulmonary aspiration have also been reported (4). Eleven cases of complete asphyxia after birth and twelve cases of severe asphyxia were registered by Nieburh (5).

Epileptic crisis is not usual (4), although sporadic cases of convulsions and spasticity have been described (7). $75 \%$ of the registered neonatal deaths occurred during the first months of life and 90\% within the first year. The most frequent causes were pneumonia, pneumonia caused by aspiration, congenital cardiac defects and respiratory distress syndrome (5).

\subsection{General manifestations}

\subsubsection{Craniofacial manifestations}

Severe mental and developmental retardation are prominent findings (below 50th and 20th percentile respectively). However no evidence has been reported to support an association with structural abnormalities of the brain.

Among the main craniofacial alterations we can emphasize microcephaly (especially evident during the first two years of life), facial dysmorphism with vertical growth pattern, severe asymmetry, moon face $(83.5 \%$ of the patients affected) that disappears in adolescence and adulthood to turn into elongated and narrow faces.
The presence of hypertelorism was pointed out by some authors, although Neibuhr considered more appropriate the term "eyes slightly separated".

Other facial alterations widely described in CdCS include the presence of epicanthal folds $(90.2 \%$ of the patients), down-turned corners of the mouth (81\%), large nasal bridge $(87.2 \%)$, short philtrum $(87.8 \%)$, convex facial profile with mandibular microretrognathia (96.7\%), abnormal dermatoglyphics $(92 \%)$ and premature grey hair $(1,3)$. Some of these aspects are showed in (Fig. $1-3)$.

Less usual findings are the presence of antimongoloid palpebral fissures $(56.9 \%)$ and low-set ears without structural anomalies added (69.8\%), preauricular tabs and narrow auditory ducts (3) (Fig. 3).

Hyperlaxitude and hypotonia in childhood eventually is replaced by hypertonia (2). Other changes associated with increase in age include the development of prominent supra-orbital arches (31\%), palpebral fissures tending to become horizontal (70.2\%) and microcephaly becoming more evident $(2,3)$.

\subsubsection{Sensitive alterations}

Several studies registered visual and auditive sensorial alterations. The most common include hypersensibility to sounds, auditive discomfort and strabismus (8). Less common alterations are deafness and hypoacusia to high

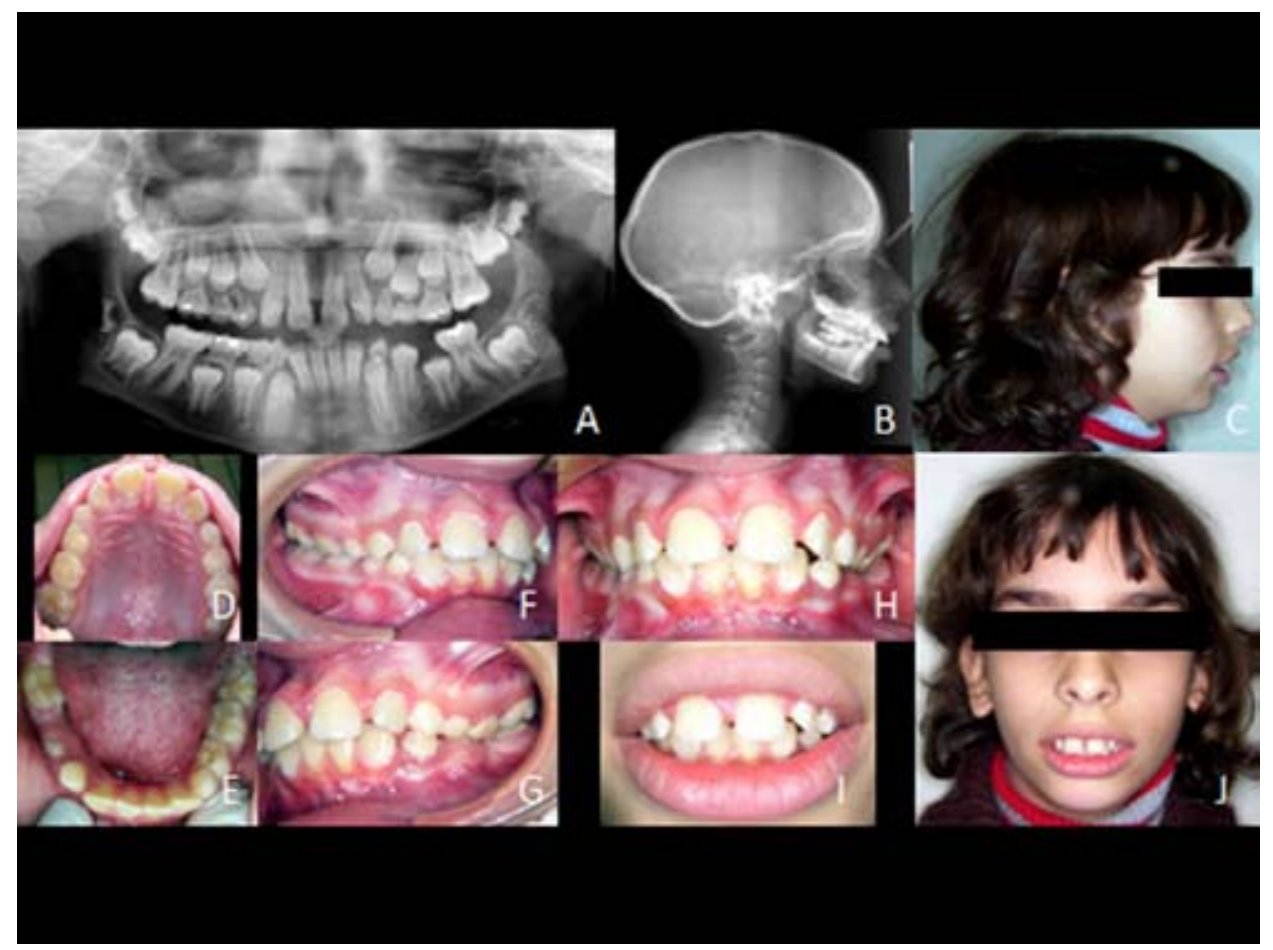

Fig. 1. A 9 year-old female, with CdCS, who presented 45 percent of handicap. Her birth was on time (40 weeks), and she weighed $3.5 \mathrm{Kg}$. She has mental retardation and scoliosis. In the orofacial area we can observe severe mandibular retrognathia and muscle hypotonia. The dental replacement was being carried out properly and she had good oral hygiene (she did not present active decay). (A. Orthopantomography - B. Lateral Cranium Radiography - C. Lateral vision of the patient - D. Occlusal vision of the upper arch - Occlusal vision of the down arch $-F$. Lateral vision of the right dental occlusion $-G$. Lateral vision of the left dental occlusion - H. Frontal vision of the dental occlusion - I. Smile - J. Frontal photography of the patient). 


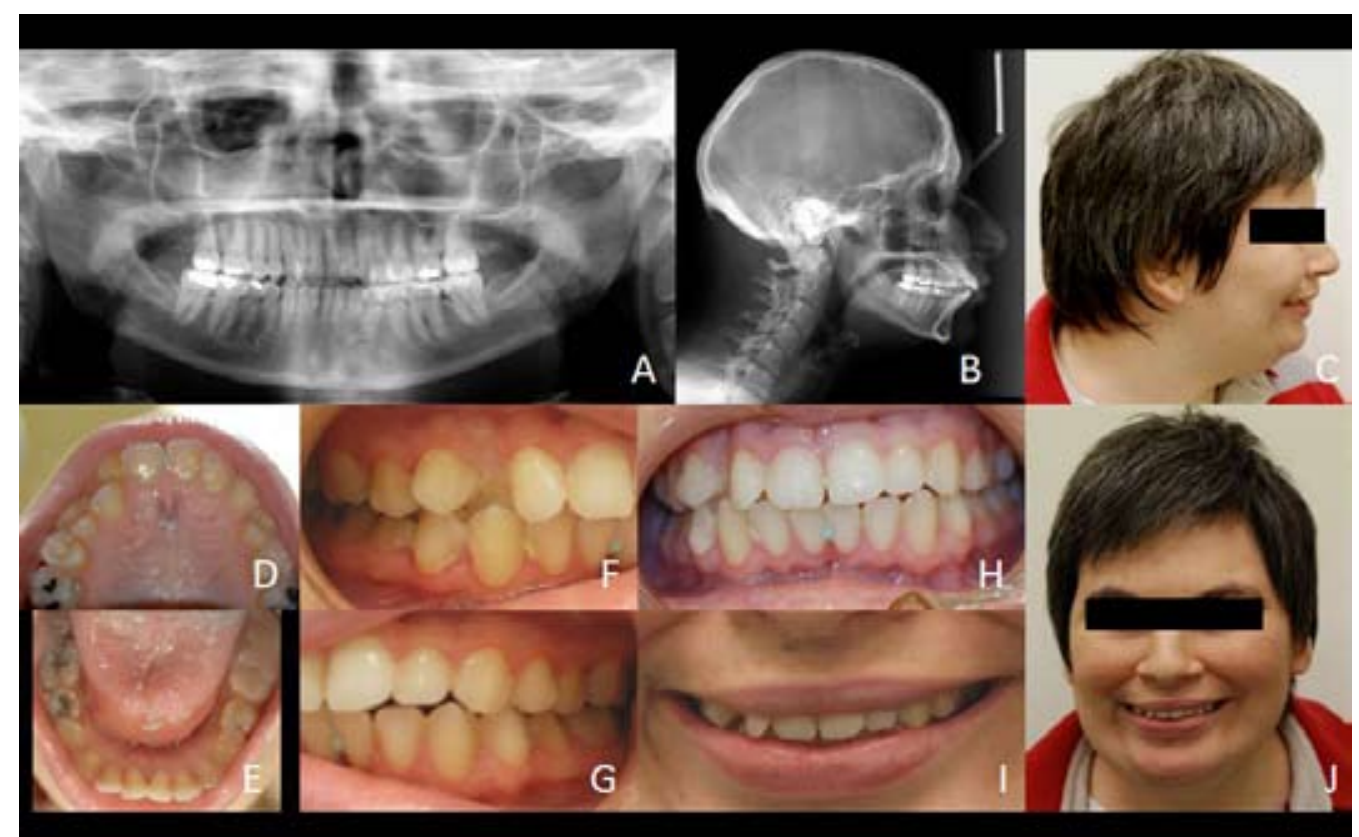

Fig. 2. A 20 year-old female, with CdCS, who presented 82 percent of handicap. Her birth was preterm (32 weeks). She weighed $2.860 \mathrm{Kg}$ and presented a cardiac ductus. Until she was 12 years of age she suffered dysphagia. She had mental retardation and moon face. The patient was intervened in order to correct her strabismus. In the orafacial area we could observe mild mandibular retrognatism. The dental replacement was being carried out properly and she had good oral hygiene (she did not present active decay). (A. Orthopantomography - B. Lateral Cranium Radiography - C. Lateral vision of the patient $-\mathrm{D}$. Occlusal vision of the upper arch - Occlusal vision of the down arch $-\mathrm{F}$. Lateral vision of the right dental occlusion - G. Lateral vision of the left dental occlusion - H. Frontal vision of the dental occlusion - I. Smile - J. Frontal photography of the patient).

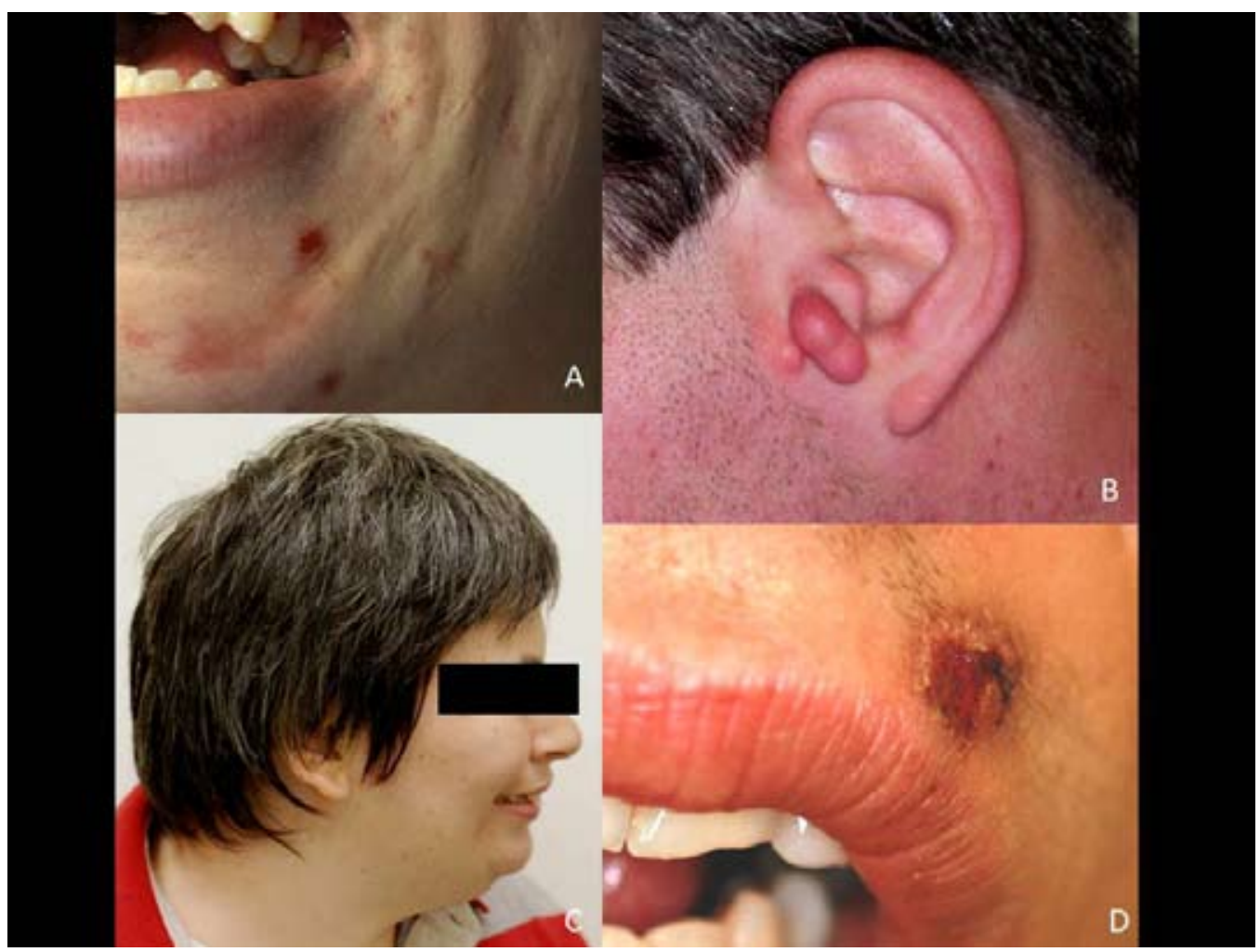

Fig. 3. Typical orofacial features of CdCS. (A, D. Perioral lesions due to self-injured behavior - B. Mild malformations (preauricular tabs) - C. Premature grey hair.) 
frequencies, myopia, cataract, hypersensitivity of the pupil to metacholine and resistence to mydriatics (probably due to a defect in the pupil dilator muscle) (8).

\subsubsection{Other anomalies}

Cardiac, skeletal, genitourinary, metabolic or immune abnormalities, although not very frequent, may be present.

Among the cardiac pathology we found cyanosis, cutaneous hemangioma, cutis marmorata, tetralogy of Fallot, cardiac congenital defects (frequently ductus associated with a septal ventricular defect and less often ductus with a septal atrial defect) $(1,3)$.

Progressive scoliosis has been frequently reported (3, 9). Patients who had increased muscular tone showed marked progression of scoliosis with growth; therefore muscular hypertonia may play a key role in the evolution of this pathology (9).

Regarding the genitourinary system, there are differences according to age. Cryptorchidism is prevalent in youth and hypogonadism in adulthood (10). Girls in pubertal age present menarche and normal sexual secondary development, but irregular menstrual periods (3). Currently there is only one case of procreation between two individuals with CdCS (11).

Renal malformations are rare, however ectopic, agenesis or horseshoe kidneys have been described.

It is possible the existence of certain metabolic anomalies such as non-cetonic hyperglycemia and deficit in the synthesis of purine (risking brain development).

An increased susceptibility to infections has been not demonstrated, however a certain alteration in granulocytosis function was detected, specifically in cellular phagocytosis.

\subsubsection{Orofacial manifestations}

The main orofacial anomalies registered were mandibular microretrognathia (75\%), high palate $(50 \%)$ but rarely often cleft $(5 \%)(7)$, variable malocclusion with anterior open-bite as the most frequent alteration (75\%) (2), enamel hypoplasia (7), poor oral hygiene, generalized chronic periodontitis, retardation of tooth eruption $(12,13)$. In spite of these alterations, there is not enough evidence to support any clear association between these pathologies and the CdCS.

It is important to point out the scarcity of published literature about patients with $\mathrm{CdCS}$ from an odontological perspective. Therefore, it is necessary to publish a wider series of cases, paying close attention to oral pathology (dental, periodontal, orthodontic and oral mucosa) in order to provide a better understanding and knowledge about it.

\section{Behavior language and Social profile}

Prior to 1989, the clinical features of CdCS were described in detail and although severe mental and developmental delay and limited communication skills were mentioned, very few articles focused on behavioral and cognitive characteristics $(1,3)$.

Currently the main behavioral problems are better defined, consisting of: hyperactivity, loss of attention, uneasiness, aggressive and self-injured behavior $(3,14)$.

Cornish and Pigram (15) found a low incidence of hyperactivity among the individuals evaluated, opposing the results that Wilkins et al. (16) obtained, that suggested hyperactivity as the major behavioral problem of more than half the population with CdCS.

As mentioned before, self-injured behavior is common (4) usually following three patterns: hitting their head against objects, hitting the head against body parts, and self-injuries caused by biting.

Hypersensitivity to sounds, repetitive movements, clumsiness, excessive happiness, stubbornness, compulsive attachment to objects and echolalia have been reported (4). All of which are potentially avoidable through proper and early educational programs.

Despite the scarcity of studies about the language skills, communication abilities and social interaction, most of the reported cases indicate that the individuals with CdCS generally have a nice and loving personality (15). Only a few cases registered show autism features or social avoidance (4).

Not all the individuals are able to develop spoken language and occasionally they use sign language with success. Invariably, receptive language and comprehension is remarkably better than expressive language (16).

Regarding the social profile, many of the children with CdCS live at home and interact with society, eventually achieving communication and coordination improvements $(15,17)$ being able to express their needs, establishing relationships with other people and developing different levels of motor activity $(4,18)$. In contrast, there are studies that suggested limited communication improvements in which a great level of individual variability is shown (17). It is still unknown whether these variations in communication skills could be attributed to phenotypic characteristics or to external factors of their environment (18).

\section{Diagnosis and Complementary tests}

Many of the clinical features of the syndrome are based on subjective assessments (moon face, hypertelorism, low-set ears, down-turned corners of the mouth, etc...) which could lead to confusion making it difficult to establish a certain clinical profile, taking into consideration that there is not a pathognomonical symptom (3). Therefore, we could conclude that a proper clinical diagnosis will depend more on the ability to recognize the combination of a collection of more or less specific situations (microcephaly, facial dismorphism, typical cry, low weight in birth, etc...) rather than on the futile seeking of the pathognomonic signs of the syndrome. 
Once the suspected diagnosis is established, the first complimentary test is a kariotype analysis. If the results are apparently normal however do not correspond with the clinic supposition, it is necessary to do more precise cytogenetic molecular analysis (FISH, CGH or quantitative PCR).

The analysis by hybridization in situ (FISH) has increased the number of diagnosis of genetic syndromes caused by a chromosomal deletion, renewing the scientific interest in CdCS because it has allowed the definition of a phenotypic map associated with the genome of the individual.

Over the last decade the FISH analysis has been complemented with other techniques, such as comparative genomic hybridization (CGH) especially the $\mathrm{CGH}$ method based on DNA microarray and quantitative PCR.

CGH is the first platform with the ability to include the whole genome with overlapping markers and it allows us to identify genetic alterations that had not been registered by other means.

\section{Prognosis and Treatment}

Both survival rate and life expectation are high, having been registered cases of individuals who were over 50 years of age. Morbi-mortality is higher during the first years of life: $75 \%$ of the registered deaths occurred during the first months after birth and $90 \%$ during the first year of life. $(2,3)$.

It is evident that the type of deletion as well as its size and location has a remarkable influence in the life prognosis of the patient, because it is directly related to the seriousness of such important alterations as mental and psychomotor delay (3).

It is necessary to distinguish $5 p$ deletions that express a typical CdCS phenotype from the ones that express an atypical clinical profile. In the former the deletion affects the critical region of the syndrome, in the latter the critical region has not been affected.

The individuals with atypical CdCS do not present a frequent severe handicap in learning (19), instead they usually present milder anomalies associated with a better prognosis. However, the individuals with typical CdCS usually present most of the characteristics associated with the syndrome and a less hopeful prognosis. We could conclude that the interstitial deletions in which the cry or language have not been affected present a better stage of development (20) than terminal deletions in which the critical region of CdCS (from 5p 15.2 to the end of the short arm) is involved.

Likewise, a more severe clinical profile is described in individuals with unbalanced translocations. In fact, in these cases mortality is considerably higher (3).

The main factor that we can manage to improve the prognosis of the patients with CdCS is the early diagnosis, because it allows us to establish early therapeutic and preventive methods oriented to promote and potentiate the physical and psychical development of the individual.

Within these methods we include carrying out the necessary surgical interventions, the early introduction of healthy routines, the integration into didactic programs and the application of rehabilitation programs. In this direction, we could alleviate behavioral disorders to increase the motor development improving their autonomy and social adaptation (2).

\section{References}

1. Lejeune J, Lafourcade J, Berger R, Vialatte J, Boeswillwald M, Seringe P, et al. [ 3 cases of partial deletion of the short arm of a 5 chromosome.]. C R Hebd Seances Acad Sci. 1963;257:3098-102.

2. Mainardi PC, Perfumo C, Calì A, Coucourde G, Pastore G, Cavani $\mathrm{S}$, et al. Clinical and molecular characterisation of 80 patients with $5 \mathrm{p}$ deletion: genotype-phenotype correlation. J Med Genet. 2001;38:151-8.

3. Niebuhr E. The Cri du Chat syndrome: epidemiology, cytogenetics, and clinical features. Hum Genet. 1978;44:227-75.

4. Cornish KM, Pigram J. Developmental and behavioural characteristics of cri du chat syndrome. Arch Dis Child. 1996;75:448-50.

5. Niebuhr E. Cytologic observations in 35 individuals with a 5pkaryotype. Hum Genet. 1978;42:143-56.

6. Colover J, Lucas M, Comley JA, Roe AM. Neurological abnormalities in the 'cri-du-chat' syndrome. J Neurol Neurosurg Psychiatry. 1972;35:711-9.

7. Sedano HO, Look RA, Carter C, Cohen MM Jr. B group short-arm deletion syndrome. Birth Defects Orig Artic Ser. 1971;7:89-97.

8. Howard RO. Ocular abnormalities in the cri du chat syndrome. Am J Ophthalmol. 1972;73:949-54.

9. Takebayashi T, Obata H, Minaki Y, Sekine M, Imoto K, Yokogushi $\mathrm{K}$, et al. Scoliosis in cat cry syndrome. J Orthop Sci. 2006;11:25963 .

10. Breg WR, Steele MW, Miller OJ, Warburton D, DeCapoa A, Allderdice PW. The cri du chat syndrome in adolescents and adults: clinical finding in 13 older patients with partial deletion of the short arm of chromosome No. 5(5p-). J Pediatr. 1970;77:782-91.

11. Martínez JE, Tuck-Muller CM, Superneau D, Wertelecki W. Fertility and the cri du chat syndrome. Clin Genet. 1993;43:212-4.

12. Scully C, Davison MF. Orofacial manifestations in the Cri du Chat syndrome (5p-). J Dent. 1979;7:313-20.

13. Boraz RA. Cri-du-chat syndrome: dental considerations and report of case. Spec Care Dentist. 1990;10:13-5.

14. Collins MS, Cornish K. A survey of the prevalence of stereotypy, self-injury and aggression in children and young adults with Cri du Chat syndrome. J Intellect Disabil Res. 2002;46:133-40.

15. Cornish KM, Pigram J. Developmental and behavioural characteristics of cri du chat syndrome. Arch Dis Child. 1996;75:44850.

16. Wilkins LE, Brown JA, Nance WE, Wolf B. Clinical heterogeneity in 80 home-reared children with cri du chat syndrome. J Pediatr. 1983;102:528-33.

17. Cornish KM, Bramble D, Munir F, Pigram J. Cognitive functioning in children with typical cri du chat (5p-) syndrome. Dev Med Child Neurol. 1999;41:263-6.

18. Kristoffersen KE. Speech and language development in cri du chat syndrome: a critical review. Clin Linguist Phon. 2008 Jun;22(6):44357. Review. Erratum in: Clin Linguist Phon. 2008;22:457.

19. Cornish K, Bramble D. Cri du chat syndrome: genotype-phenotype correlations and recommendations for clinical management. Dev Med Child Neurol. 2002;44:494-7.

20. Church DM, Yang J, Bocian M, Shiang R, Wasmuth JJ. A highresolution physical and transcript map of the Cri du chat region of human chromosome 5p. Genome Res. 1997;7:787-801. 